\title{
On the Second-order Temperature Jump Coefficient of a Dilute Gas
}

\author{
Gregg A. Radtke and Nicolas G. Hadjiconstantinou* \\ Mechanical Engineering Department, Massachusetts Institute of Technology \\ Cambridge, MA 02139, U.S.A. \\ Shigeru Takata and Kazuo Aoki \\ Department of Mechanical Engineering and Science, Kyoto University \\ Kyoto 606-8501, Japan
}

August 20, 2018

\begin{abstract}
We use LVDSMC simulations to calculate the second-order temperature jump coefficient for a dilute gas whose temperature is governed by the Poisson equation with a constant forcing term. Both the hard sphere gas and the BGK model of the Boltzmann equation are considered. Our results show that the temperature jump coefficient is different from the well known linear and steady case where the temperature is governed by the homogeneous heat conduction (Laplace) equation.
\end{abstract}

\section{Introduction}

Slip-flow theory is a powerful tool that enables the continued use of the Navier-Stokes description as the characteristic flow lengthscale $(L)$ approaches the molecular mean free path $(\lambda)$ [14]. It can be rigorously derived from asymptotic solution of the Boltzmann equation in the limit $K n=\lambda / L \ll 1$; such an analysis shows that, in this limit, the Navier-Stokes description remains valid in the bulk, but fails near the boundaries [16, 17]. Fortunately, the kinetic effects associated with the inhomogeneity introduced by the walls are only important within a layer of thickness $O(\lambda)$ near the boundaries (known as the Knudsen layer) and can be accounted for by a boundary-layer type of analysis where an inner kinetic solution is matched to the outer Navier-Stokes solution [16, 17]. Slip/jump boundary conditions and the associated non-adjustable slip coefficients emerge from this analysis as the matching

*Author to whom correspondence should be addressed. Electronic mail: ngh@mit.edu 
condition between the inner and outer solution [16, 17]. Carrying out such an analysis to second order in $K n$ yields second-order slip/jump models [16, 17], which can be very useful in a variety of cases [14].

Accurate determination of slip coefficients using this rigorous procedure is quite challenging in general and becomes increasingly more challenging as the order of the expansion increases. Original studies focused on the BGK model of the Boltzmann equation [3, 6], for which all first-order and second-order coefficients are known [16, 17]. The first-order coefficients for the hard-sphere gas have also since been calculated [5]. However, although the form of the slip expression is known to second order in $K n$, second-order slip coefficients for the hard-sphere gas are mostly unknown.

As the companion paper shows [7], the recently developed reciprocity relations by Takata [1, 2] can be used to calculate these coefficients. An alternative approach amounts to extracting slip coefficients from hydrodynamic fields by comparing solutions of the Boltzmann equation with Navier-Stokes solutions [8, 14]. In these approaches, in addition to high accuracy (including low statistical uncertainty if a stochastic method is used for solving the Boltzmann equation), care needs to be exercised to avoid comparison of the two solutions in the Knudsen layer, where the Navier-Stokes solution is not equivalent to the Boltzmann solution [14]. This has led to a number of erroneous results in the past.

In this paper we use this process to calculate the second-order temperature jump coefficient for a dilute gas when the temperature field is governed by the Poisson equation with constant forcing term. We calculate this coefficient using the recently developed low-variance deviational Monte Carlo simulation method [12, 13, 15, 18, 9], which is naturally suited to low-signal problems and thus allows calculations at infinitessimal temperature differences. The latter are necessary because finite temperature differences introduce density gradients and temperature-dependent transport coefficients which may alter the result.

Our result is verified and put on a more firm theoretical footing by the companion paper [7] which considers a mathematically equivalent time-dependent problem, thus clarifying why the temperature jump law and coefficient reported here are in general different from the one obtained by linear steady-state analysis [16].

\section{Background}

We consider a dilute hard-sphere gas of molecular mass $m$ and molecular diameter $\sigma$, in contact with a planar diffusely reflecting boundary at temperature $T_{\mathrm{B}}$. We also consider the BGK model of such a gas, with collision frequency $\tau^{-1}$. In the case of the hard-sphere gas, $\lambda=\left(\sqrt{2} \pi n_{0} \sigma^{2}\right)^{-1}$, while for the BGK gas $\lambda=2 c_{0} \tau / \sqrt{\pi}$, where $c_{0}=\sqrt{2 R T_{0}}$ is the most probable speed based on the reference temperature $T_{0}, n_{0}$ is a reference number density, $R=k_{B} / m$ is the gas constant and $k_{B}$ is Boltzmann's constant.

The first-order temperature jump condition at the gas-wall interface is given by [16, 17]

$$
\left.\hat{T}\right|_{\mathrm{B}}-\hat{T}_{\mathrm{B}}=\left.d_{1} k \frac{\partial \hat{T}}{\partial \hat{n}}\right|_{\mathrm{B}}
$$


where $\hat{T}=T / T_{0}, k=\frac{\sqrt{\pi}}{2} K n=\frac{\sqrt{\pi}}{2}(\lambda / L)$, $\left.\right|_{\mathrm{B}}$ denotes the boundary location, $\hat{n}$ is the unit (inward) normal direction and $L$ is the characteristic system length scale; the numerical constant $d_{1}$ obtains the non-adjustable values of 2.4001 for a hard sphere gas and 1.30272 for a BGK gas [16]; we emphasize that these values correspond to diffusely-reflecting boundaries.

The utility of first-order slip/jump models primarily depends on the amount of error that can be tolerated. Temperature jump coefficients (both first-order and the second-order measured here) turn out to be larger than their velocity slip counterparts. As a result, a second-order temperature jump correction becomes important at smaller Knudsen numbers. In other words, the first-order result (1) is typically adequate for $K n<0.1$.

Asymptotic expansion to second order in $k$ [16, 17] for linear and steady problems extends (1) to the following jump condition

$$
\left.\hat{T}\right|_{\mathrm{B}}-\hat{T}_{\mathrm{B}}=\left.d_{1} k \frac{\partial \hat{T}}{\partial \hat{n}}\right|_{\mathrm{B}}+\left.d_{3} k^{2} \frac{\partial^{2} \hat{T}}{\partial \hat{n}^{2}}\right|_{\mathrm{B}} .
$$

This condition is valid for a quiescent gas - more precisely, a gas that is quiescent under no-slip boundary conditions; in the presence of gas flow, additional terms related to the flow stress need to be included [16]. We also emphasize that according to the analysis that yields this condition, for linear and steady problems, energy conservation reduces to

$$
\nabla^{2} \hat{T}=\frac{\partial^{2} \hat{T}}{\partial \hat{x}^{2}}+\frac{\partial^{2} \hat{T}}{\partial \hat{y}^{2}}+\frac{\partial^{2} \hat{T}}{\partial \hat{z}^{2}}=0,
$$

where $(\hat{x}, \hat{y}, \hat{z})=(x / L, y / L, z / L)$, and $L$ is the characteristic problem lengthscale. In the special case of one-dimensional problems, equation (3) further reduces to

$$
\nabla^{2} \hat{T}=\frac{\mathrm{d}^{2} \hat{T}}{\mathrm{~d} \hat{n}^{2}}=0
$$

which makes the value of $d_{3}$ irrelevant. This is actually utilized below to calculate the slip coefficient due to a forcing term in the temperature equation.

In summary, jump condition (2) is to be used when the governing equation is (3). Within this approximation, $d_{3}$ is only known $\left(d_{3}=0\right)$ for the special case of the BGK model [16, 17]. We also note that Deissler's result [4] for second-order velocity slip and temperature jump is based on approximate mean-free-path arguments and does not correspond to a self-consistent solution of the Boltzmann equation; as a result, it captures neither the correct form of the slip/jump relation nor the correct values of the slip coefficients (e.g. compare equations (3.40)-(3.42) in [17] to equations (24a) and (51) in [4]).

\section{Calculation of the temperature jump coefficient}

To extract the slip coefficient in a dilute gas governed by the Poisson equation with constant forcing term, we simulate the steady state of a one-dimensional gas layer bounded by two isothermal, diffuse walls at $x= \pm L / 2$ and at temperature $T_{0}$, subject to volumetric heating 
at a constant rate $\dot{Q}$. In dimensionless form, the one-dimensional heat equation with constant volumetric heating can be written as

$$
\nabla^{2} \hat{T}=\frac{\mathrm{d}^{2} \hat{T}}{\mathrm{~d} \hat{x}^{2}}=-\frac{5 \epsilon}{4 \gamma_{2} k},
$$

where $\hat{x}=x / L, \gamma_{2}$ is a dimensionless form of the thermal conductivity- equal to 1.9228 for hard spheres and unity for BGK [17] - and

$$
\epsilon=\frac{L \dot{Q}}{c_{0} P_{0}} \ll 1
$$

is the dimensionless form of the volumetric heat addition rate. Here, $P_{0}=n_{0} k_{B} T_{0}$ is a reference pressure.

The asymptotic analysis yielding (2) does not apply to the non-homogeneous equation (5). A rigorous derivation which takes the inhomogeneous term into account by considering an equivalent unsteady problem can be found in the companion paper [7], which shows that in a quiescent gas, in one spatial dimension, the resulting second-order slip relation is given by

$$
\left.\hat{T}\right|_{\mathrm{B}}-\hat{T}_{\mathrm{B}}=\left.d_{1} k \frac{\partial \hat{T}}{\partial \hat{n}}\right|_{\mathrm{B}}+\left.d_{3}^{\prime} k^{2} \frac{\partial^{2} \hat{T}}{\partial \hat{n}^{2}}\right|_{\mathrm{B}} \cdot
$$

We emphasize that, although the structure of the slip relation is the same as in equation (2), the second-order coefficient is different. It is also convenient that (7) does not contain $d_{3}$; this allows calculation of $d_{3}^{\prime}$ from volumetric heating calculations without explicit knoweldge of $d_{3}$. This last feature, as well as the similarity of (2) and (7) is due to fortuitous cancellation; as discussed further in section 6, under more general conditions (e.g. higher spatial dimensions), this cancellation does not take place and terms containing both $d_{3}$ and $d_{3}^{\prime}$ appear.

The solution to Equation (5) subject to boundary condition (7) is

$$
\hat{T}=\frac{1}{2} \frac{4 \epsilon}{5 \gamma_{2} k}\left[\left(\frac{1}{4}-\hat{x}^{2}\right)+d_{1} k-2 d_{3}^{\prime} k^{2}\right] .
$$

Comparison of this solution to LVDSMC simulations away from the Knudsen layer allows us to calculate the coefficient $d_{3}^{\prime}$. In this work, we extract the value of $d_{3}^{\prime}$ from the slope of

$$
\frac{5 \gamma_{2}}{4 \epsilon} \hat{T}(\hat{x}=0)-\frac{1}{8 k}-\frac{d_{1}}{2}
$$

as a function of $k$ for $k \rightarrow 0$.

\section{Computational method}

The Low-variance Deviational Simulation Monte Carlo (LVDSMC) method [12, 13, 15, 18, 9] efficiently simulates [10] the Boltzmann equation

$$
\frac{\partial f}{\partial t}+\boldsymbol{c} \cdot \frac{\partial f}{\partial \boldsymbol{x}}=\left[\frac{\partial f}{\partial t}\right]_{\mathrm{coll}}
$$


written here in the absence of external body forces, by simulating only the deviation $f^{\mathrm{d}}=$ $f-f^{\mathrm{MB}}$ from an equilibrium state $f^{\mathrm{MB}}$. Here, $f=f(\boldsymbol{x}, \boldsymbol{c}, t)$ is the single particle distribution function [16]. This approach results in a greatly reduced level of statistical uncertainty for low signal problems compared to the standard DSMC [11] approach and is therefore well suited to the present application.

Volumetric heating is modeled by simulating the equation

$$
\frac{\partial f}{\partial t}+\boldsymbol{c} \cdot \frac{\partial f}{\partial \boldsymbol{x}}=\left[\frac{\partial f}{\partial t}\right]_{\text {coll }}+\frac{\dot{Q}}{P_{0}}\left(\frac{2}{3} \frac{c^{2}}{c_{0}^{2}}-1\right) f^{0}
$$

where

$$
f^{0}=\frac{\rho_{0}}{\pi^{3 / 2} c_{0}^{3}} e^{-\|\boldsymbol{c}\|^{2} / c_{0}^{2}}
$$

and $\rho_{0}=m n_{0}$ is a reference mass density. More details on the simulation of the additional term on the right hand side can be found in section 4.2 .

In the versions implemented here, equilibrium is described by a Maxwell-Boltzmann distribution

$$
f^{\mathrm{MB}}=\frac{\rho_{\mathrm{MB}}}{\pi^{3 / 2} c_{\mathrm{MB}}^{3}} \exp \left(-\frac{\left\|\boldsymbol{c}-\boldsymbol{u}_{\mathrm{MB}}\right\|^{2}}{c_{\mathrm{MB}}^{2}}\right),
$$

based on local (cell-based) mass density $\rho_{\mathrm{MB}}$, velocity $\boldsymbol{u}_{\mathrm{MB}}$, temperature $T_{\mathrm{MB}}$, and most probable velocity $c_{\mathrm{MB}}=\sqrt{2 R T_{\mathrm{MB}}}$. Because $f^{\mathrm{d}}$ can take positive and negative values, it is represented by signed (or deviational) particles.

As in the DSMC approach, LVDSMC solves the Boltzmann transport equation through a time-splitting approach using a timestep $\Delta t$. The associated advection and collision substeps are described below.

\subsection{Advection substep}

During the advection substep, particles move according to the standard DSMC procedure (i.e. for particle $\left.i, \boldsymbol{x}_{i}(t+\Delta t)=\boldsymbol{x}_{i}(t)+\boldsymbol{c}_{i} \Delta t\right)$, with additional particles generated at the boundaries and cell interfaces. Each of these additional generation steps are implemented by drawing particles from differences of fluxal distributions.

At a stationary boundary, particles are generated by sampling from

$$
\boldsymbol{c} \cdot \boldsymbol{n}\left(\rho_{\mathrm{B}} \phi^{\mathrm{B}}-f^{\mathrm{MB}}\right) \Delta A \Delta t \mathrm{~d}^{3} \boldsymbol{c},
$$

where $\Delta A$ is the surface area element at the boundary, $f^{\mathrm{MB}}$ is the equilibrium distribution in the cell adjacent to the boundary, and $\phi^{\mathrm{B}}$ is the "boundary distribution" given by

$$
\phi^{\mathrm{B}}=\frac{e^{-c^{2} / c_{\mathrm{B}}^{2}}}{\pi^{3 / 2} c_{\mathrm{B}}^{3}},
$$

where the $c_{\mathrm{B}}=\sqrt{2 R T_{\mathrm{B}}}$; the "boundary density" $\rho_{\mathrm{B}}$ is evaluated from the mass conservation statement

$$
\rho_{\mathrm{B}} \int_{\boldsymbol{c} \cdot \boldsymbol{n}>0} \boldsymbol{c} \cdot \boldsymbol{n} \phi^{\mathrm{B}} \mathrm{d}^{3} \boldsymbol{c}=-\int_{\boldsymbol{c} \cdot \boldsymbol{n}<0} \boldsymbol{c} \cdot \boldsymbol{n} f^{\mathrm{MB}} \mathrm{d}^{3} \boldsymbol{c} .
$$


Particles are also generated at the cell interfaces to account for the spatial discontinuities in $f^{\mathrm{MB}}[13,15]$; they are sampled from

$$
\boldsymbol{c} \cdot \boldsymbol{n}\left(f_{-}^{\mathrm{MB}}-f_{+}^{\mathrm{MB}}\right) \Delta A^{\mathrm{int}} \Delta t \mathrm{~d}^{3} \boldsymbol{c}
$$

where $\Delta A^{\mathrm{int}}$ is the area of the interface, $f_{ \pm}^{\mathrm{MB}}$ are the equilibrium distributions in adjacent cells, and $\boldsymbol{n}$ points from $f_{-}^{\mathrm{MB}}$ to $f_{+}^{\mathrm{MB}}$.

\subsection{Collision substep}

The collision substep treatment is based on published LVDSMC implementations [15, 9], suitably modified to include the effect of volumetric heating. We first discuss the BGK collision operator and the corresponding volumetric heating implementation; the hard-sphere case follows. Due to the small deviations from equilibrium, here we consider the linearized form of these collision operators; methods for simulating the corresponding non-linear versions can be found in [18, 13, 10].

\subsubsection{BGK model}

In the case of the BGK model, the collision operator is given by

$$
\left[\frac{\partial f}{\partial t}\right]_{\text {coll }}=-\frac{f-f^{\mathrm{loc}}}{\tau}
$$

where $f^{\text {loc }}$ is the local equilibrium distribution given by

$$
f^{\text {loc }}=\frac{\rho(\boldsymbol{x}, t)}{[2 \pi R T(\boldsymbol{x}, t)]^{3 / 2}} \exp \left(-\frac{\|\boldsymbol{c}-\boldsymbol{u}(\boldsymbol{x}, t)\|^{2}}{2 R T(\boldsymbol{x}, t)}\right),
$$

where $\rho(\boldsymbol{x}, t), \boldsymbol{u}(\boldsymbol{x}, t)$ and $T(\boldsymbol{x}, t)$ are the local mass density, flow velocity and temperature.

Using the approach of Ref. [15], the collision step for the BGK collision operator is written as

$$
\left[\frac{\partial f^{\mathrm{d}}}{\partial t}\right]_{\text {coll }} \Delta t=\underbrace{\frac{\Delta t}{\tau}\left[f^{\mathrm{loc}}-f^{\mathrm{MB}}\right]-\Delta f^{\mathrm{MB}}}_{\text {generation }}-\underbrace{\frac{\Delta t}{\tau} f^{\mathrm{d}}}_{\text {deletion }},
$$

where $\Delta f^{\mathrm{MB}}$ is a shift in the equilibrium state. The terms above represent a source term for generating new particles, and a sink term for deleting existing particles. It can be shown [15] that the generation term is eliminated for linear problems when the equilibrium state (for each cell) is shifted according to

$$
\left[\begin{array}{c}
\rho_{\mathrm{MB}} \\
\boldsymbol{u}_{\mathrm{MB}} \\
T_{\mathrm{MB}}
\end{array}\right](t+\Delta t)=\left[\begin{array}{c}
\rho_{\mathrm{MB}} \\
\boldsymbol{u}_{\mathrm{MB}} \\
T_{\mathrm{MB}}
\end{array}\right](t)+\frac{\Delta t}{\tau}\left[\begin{array}{c}
\rho-\rho_{\mathrm{MB}} \\
\boldsymbol{u}-\boldsymbol{u}_{\mathrm{MB}} \\
T-T_{\mathrm{MB}}
\end{array}\right](t) .
$$

This results in a substantial simplification to step (20), which reduces to the very simple operation of randomly deleting particles with probability $\Delta t / \tau$. 
Because the above method simulates a local equilibrium $f^{\mathrm{MB}}$ that is updated in the course of the simulation, the heat generation term can be introduced directly (and analytically) into the algorithm using

$$
\dot{Q}=\rho_{0} \frac{\mathrm{d}}{\mathrm{d} t}\left(\frac{3}{2} R T_{M B}\right),
$$

which results in the following update for the temperature parameter of the equilibrium distribution

$$
\Delta T_{\mathrm{MB}}=\frac{2 \dot{Q} \Delta t}{3 \rho_{0} R}
$$

every timestep.

\subsubsection{Hard Sphere model}

The hard sphere collision operator is given by

$$
\left[\frac{\partial f}{\partial t}\right]_{\text {coll }}=\frac{1}{m} \iint\left(f^{\prime} f_{*}^{\prime}-f f_{*}\right) \frac{\sigma^{2}}{4}\left\|\boldsymbol{c}-\boldsymbol{c}_{*}\right\| \mathrm{d}^{3} \boldsymbol{c}_{*} \mathrm{~d}^{2} \boldsymbol{\Omega},
$$

where primes denote post-collision values and $\Omega$ is the spherical angle. The collision step for this approach

$$
\left[\frac{\partial f^{\mathrm{d}}}{\partial t}\right]_{\text {coll }}=\underbrace{\int\left[2 K^{(1)}-K^{(2)}\right]\left(\boldsymbol{c}, \boldsymbol{c}_{*}\right) f_{*} \mathrm{~d}^{3} \boldsymbol{c}}_{\text {generation }}-\underbrace{\nu f}_{\text {deletion }}
$$

is processed as a series of Markov particle generation and deletion steps as proposed by Wagner [10]; the specific algorithms employed are discussed in detail in Refs. [10, 9]. In the above,

$$
\begin{aligned}
K^{(1)}\left(\boldsymbol{c}, \boldsymbol{c}_{*}\right) & =\frac{\sigma^{2} \rho_{\mathrm{MB}}}{\sqrt{\pi} m c_{\mathrm{MB}}\left\|\boldsymbol{c}-\boldsymbol{c}_{*}\right\|} \exp \left(-\frac{\left[\left(\boldsymbol{c}-\boldsymbol{u}_{\mathrm{MB}}\right) \cdot\left(\boldsymbol{c}-\boldsymbol{c}_{*}\right)\right]^{2}}{c_{\mathrm{MB}}^{2}\left\|\boldsymbol{c}-\boldsymbol{c}_{*}\right\|^{2}}\right) \\
K^{(2)}\left(\boldsymbol{c}, \boldsymbol{c}_{*}\right) & =\frac{\pi \sigma^{2}}{m}\left\|\boldsymbol{c}-\boldsymbol{c}_{*}\right\| f^{\mathrm{MB}}(\boldsymbol{c}) \\
\nu(\boldsymbol{c}) & =\frac{\pi \sigma^{2} \rho_{\mathrm{MB}} c_{\mathrm{MB}}}{m}\left[\frac{e^{-\xi^{2}}}{\sqrt{\pi}}+\left(\xi+\frac{1}{2 \xi}\right) \operatorname{erf}(\xi)\right]
\end{aligned}
$$

where $\xi=\left\|\boldsymbol{c}-\boldsymbol{u}_{\mathrm{MB}}\right\| / c_{\mathrm{MB}}$.

In this approach, $f^{\mathrm{MB}}$ is not updated during the collision step because the hard-sphere simulation algorithm used here is based [9, 19] on the fixed global equilibrium distribution $f^{0}$. However, to improve accuracy for the low values of $K n$ considered here ${ }^{1}$ we have developed

\footnotetext{
${ }^{1}$ As shown in [15], due to the increasing importance of the local equilibrium distribution as $K n \rightarrow 0$, LVDSMC simulations with a variable equilibrium distribution significantly outperform their counterparts with fixed equilibrium distribution, because they can be set up to track the local equilibrium distribution and thus minimize the number of particles required for the same solution fidelity.
} 
a special algorithm which uses an equilibrium distribution $\left(f^{\mathrm{MB}}\right)$ that is not updated during the collision step but is, however, spatially dependent. In order to determine a suitable form of $f^{M B}$, at the early stages of the simulation, this distribution tracks the local equilibrium distribution (similarly to the BGK algorithm described above) using an iterative algorithm in which $\rho_{\mathrm{MB}}$ and $T_{\mathrm{MB}}$ are taken from the solution at the previous iteration, while the velocity $\boldsymbol{u}_{\mathrm{MB}}$ is taken to be zero. This process is started with $f^{\mathrm{MB}}=f^{0}$ and iterated until $f^{\mathrm{MB}}$ no longer changes appreciably, which usually takes less than 2 iterations.

The uniform heat generation is implemented in this case by generating particles from the distribution

$$
\left[\frac{\partial f^{\mathrm{d}}}{\partial t}\right]_{\text {heat }}=\frac{\dot{Q}}{P_{0}}\left(\frac{2}{3} \frac{c^{2}}{c_{0}^{2}}-1\right) f^{0} .
$$

Algorithms for efficiently sampling from distributions of the form (29) are described elsewhere [15, 9, 19].

\section{$5 \quad$ Results}

Numerical simulations of the uniform heat generation problem were performed in order to extract the second-order jump coefficients by comparing the calculated steady centerline temperature $\hat{T}(\hat{x}=0)$ with the prediction of equation $(8)$ at $\hat{x}=0$.

Figure 1 shows our numerical data for $-k d_{3}^{\prime}$ and a linear least squares fit passing through the origin based on the data for $k<0.06$, and the values $d_{1}=1.30272$ for BGK and $d_{1}=2.4001$ for the hard sphere gas [17]. These fits yield $d_{3}^{\prime}=-1.4$ for BGK and -3.1 for the hard sphere model; the fit quality demonstrates that the leading order term is indeed $k^{2}$. The contribution of higher order terms starts to be noticable as $k$ increases. Incidentally, the complementary analysis of the companion paper [7], based on a finite difference analysis of the Knudsen-layer problem of the linearized Boltzmann equation, yields $d_{3}^{\prime}=-1.4276$ for the BGK model and $d_{3}^{\prime}=-3.180$ for the hard-sphere model.

Figure 2 shows the temperature field for the hard sphere case with $K n=0.05$ (equivalent to $k=0.0443$ ) using the value obtained above (namely $d_{3}^{\prime}=-3.1$ ) demonstrating excellent agreement everywhere except in the Knudsen layer close to the boundary, as expected. By comparing the first- and second-order jump theories, it is clear that the second-order jump theory provides an improvement over the existing first-order theory, already at $K n=0.05$. For $K n=0.1$ (Figure 3), the error in the first order solution is quite large, while the secondorder solution is considerably more accurate, provided that the existence of the Knudsen layers for a large part of the domain is accounted for.

\section{Discussion}

Using LVDSMC simulations, we have extracted the second-order temperature jump coefficient for a hard-sphere and a BGK gas in the case that the Navier-Stokes-limit behavior is captured by an inhomogeneous heat conduction equation, such as the one appearing in 


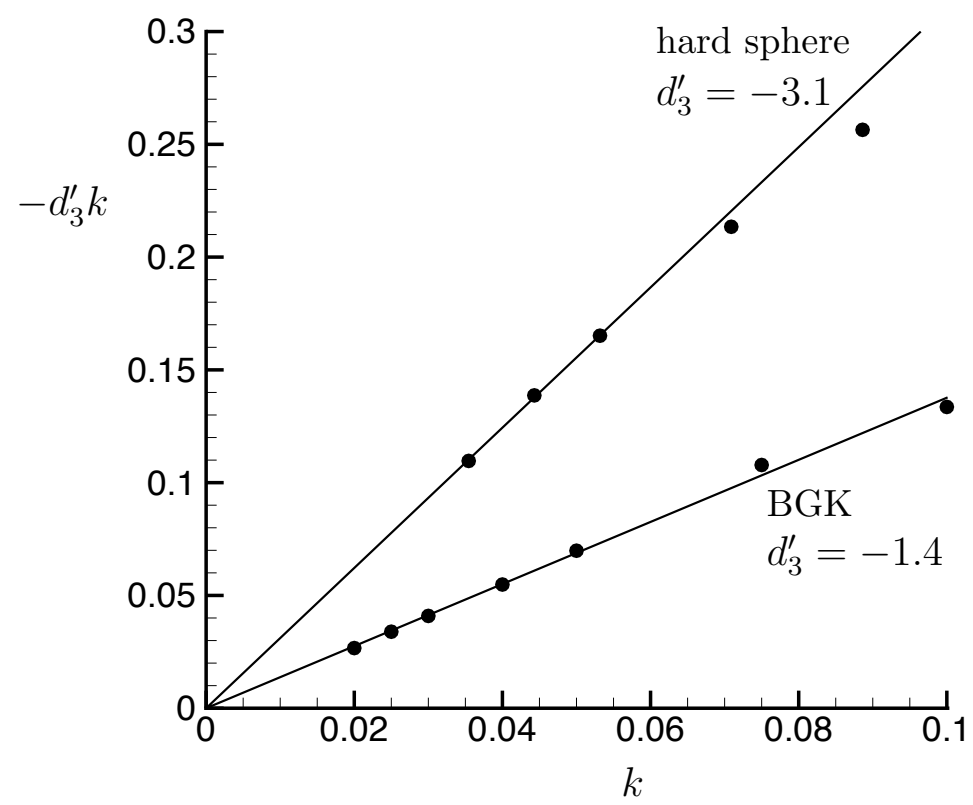

Figure 1: Fits used to extract the second-order jump coefficient $d_{3}^{\prime}$ for the hard sphere and BGK collision models.

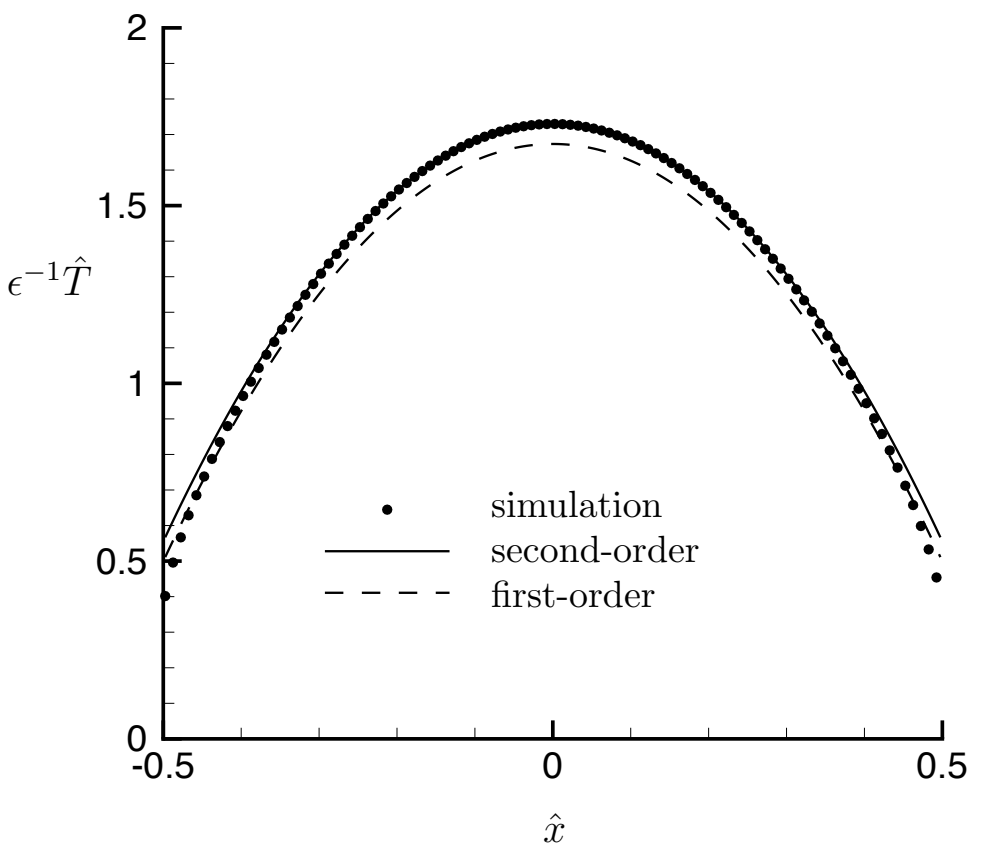

Figure 2: Second-order temperature jump solution (Equation (8)) to the uniform heat generation problem with Knudsen number $K n=0.05$; simulation results (symbols) are compared to the first- (dashed line) and second-order (solid line) jump theories (equation (8) with $d_{3}^{\prime}=0$ and $d_{3}^{\prime}=-3.1$, respectively). 


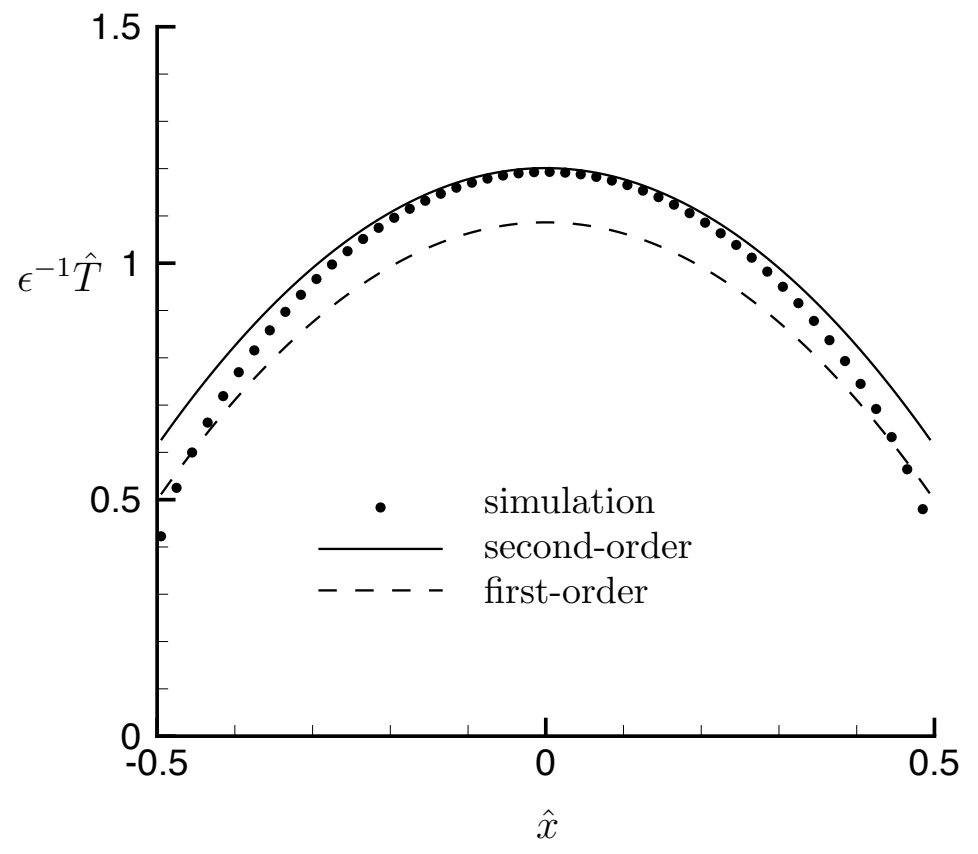

Figure 3: Second-order temperature jump solution (Equation (8)) to the uniform heat generation problem with Knudsen number $K n=0.1$; simulation results (symbols) are compared to the first-order (dashed line) and second-order (solid line) jump theories (equation (8) with $d_{3}^{\prime}=0$ and $d_{3}^{\prime}=-3.1$, respectively). 
the presence of constant volumetric heating. Our results have been validated by a companion paper which provides a deterministic calculation of the same coefficient through a rigorous asymptotic analysis of the Boltzmann description of a mathematically equivalent problem, namely that of a quescient gas confined between two parallel walls whose temperature changes linearly (increases or decreases) in time at a constant (and small) rate. Due to the time-dependent nature of the latter problem, the analysis in the companion paper goes beyond the asymptotic theory for steady problems [16]; this also explains why the presently calculated jump coefficient $\left(d_{3}^{\prime}\right)$ is not equivalent to the one $\left(d_{3}\right)$ obtained by the steady asymptotic analysis of Ref. [16].

Equation (5) and boundary condition (7) can be generalized to two and three-dimensional steady problems as long as the heat generation in the gas is uniform in space and constant in time. Specifically, for a quiescent gas, the governing equation and boundary condition in this case become

$$
\nabla^{2} \hat{T}=-\frac{5 \epsilon}{4 \gamma_{2} k}
$$

and

$$
\begin{aligned}
&\left.\hat{T}\right|_{\mathrm{B}}-\hat{T}_{\mathrm{B}}=\left(d_{1}+d_{5} \bar{\kappa} k\right)\left.k \frac{\partial \hat{T}}{\partial \hat{n}}\right|_{\mathrm{B}}+\left.d_{3}^{\prime} k^{2} \frac{\partial^{2} \hat{T}}{\partial \hat{n}^{2}}\right|_{\mathrm{B}} \\
&+\left.\left(d_{3}^{\prime}-d_{3}\right) k^{2}\left(\nabla^{2} \hat{T}-\frac{\partial^{2} \hat{T}}{\partial \hat{n}^{2}}\right)\right|_{\mathrm{B}}
\end{aligned}
$$

respectively. Here $\bar{\kappa} / L$ is the mean boundary curvature and $d_{5}=1.82181$ for the BGK model [16]; for the hard-sphere gas the value of $d_{5}$ is unknown. The temperature jump coefficient $d_{3}$ for the hard sphere gas, as well as a general second-order slip description of unsteady problems remain unknown and will be the subject of future work.

\section{Acknowledgements}

This work was supported in part by the Singapore-MIT Alliance. NGH would like to thank KA for his hospitality during his visit to Kyoto University in 2010.

\section{References}

[1] S. Takata, "Symmetry of the linearized Boltzmann equation and its application", J. Stat. Phys., 136, 751, 2009.

[2] S. Takata, "Symmetry of the unsteady linearized Boltzmann equation in a fixed bounded domain", J. Stat. Phys., 140, 985, 2010.

[3] C. Cercignani, "Elementary solutions of the linearized gasdynamics Boltzmann equation and their application to the slip-flow problem", Ann. Phys., 20, 219, 1962. 
[4] R. G. Deissler, "An analysis of second-order slip flow and temperature-jump boundary conditions for rarefied gases", Int. J. Heat Mass Transfer, 7, 681-694, 1964.

[5] Y. Sone, T. Ohwada and K. Aoki, "Temperature jump and Knudsen layer in a rarefied gas over a plane wall: Numerical analysis of the linearized Boltzmann equation for hard-sphere molecules", Phys. Fluids A, 1, 363, 1989; T. Ohwada Y. Sone and K. Aoki, "Numerical analysis of the shear and thermal creep flows of a rarefied gas over a plane wall on the basis of the linearized Boltzmann equation for hard-sphere molecules", Phys. Fluids A, 1, 1588, 1989; T. Ohwada Y. Sone and K. Aoki, "Numerical analysis of the Poiseuille and thermal transpiration flows between parallel plates on the basis of the Boltzmann equation for hard-sphere molecules", Phys. Fluids A, 1, 2042, 1989.

[6] Y. Sone, "Asymptotic theory of flow of rarefied gas over a smooth boundary I", in Rarefied Gas Dynamics, Proceedings of the Sixth International Symposium on Rarefied Gas Dynamics, edited by L. Trilling and H. Y. Wachman, Vol. 1, pp.243-253, Academic Press, NY, 1969; Y. Sone, "Asymptotic theory of flow of rarefied gas over a smooth boundary II", in Rarefied Gas Dynamics, Proceedings of the Seventh International Symposium on Rarefied Gas Dynamics, edited by D. Dini, Vol. 2, pp.737-749, Editrice Tecnico Scientifica, Pisa, 1971.

[7] S. Takata, K. Aoki, M. Hattori and N.G. Hadjiconstantinou, "Parabolic temperature profile and second-order temperature jump of a slightly rarefied gas in an unsteady two-surface problem", companion paper.

[8] N. G. Hadjiconstantinou, "Comment on Cercignani's second-order slip coefficient", Phys. Fluids, 15, 2352-2354, 2003.

[9] G. A. Radtke, N. G. Hadjiconstantinou and W. Wagner, "Low-noise Monte Carlo Simulation of the Variable Hard-sphere Gas", Physics of Fluids, 23, 030606, 2011.

[10] W. Wagner, "Deviational particle Monte Carlo for the Boltzmann equation", Monte Carlo Methods Appl., 14, 191-268, 2008.

[11] G. A. Bird, Molecular Gas Dynamics and the Direct Simulation of Gas Flows, Oxford Science, 1994

[12] T. M. M. Homolle, N.G. Hadjiconstantinou, "Low-variance deviational simulation Monte Carlo", Phys. Fluids, 19, 041701, 2007.

[13] T. M. M. Homolle and N. G. Hadjiconstantinou, "A Low-variance deviational simulation Monte Carlo for the Boltzmann equation", J. Comp. Phys., 226, 2341-2358, 2007.

[14] N. G. Hadjiconstantinou, "The limits of Navier-Stokes theory and kinetic extensions for describing small-scale gaseous hydrodynamics", Phys. Fluids, 18(11): 111301, 2006. 
[15] G. A. Radtke and N. G. Hadjiconstantinou, "Variance-reduced particle simulation of the Boltzmann transport equation in the relaxation-time approximation", Phys. Rev. E 79 (5): 056711, 2009.

[16] Y. Sone, Kinetic Theory and Fluid Dynamics, Birkhauser, Boston, 2002.

[17] Y. Sone, Molecular Gas Dynamics: Theory, Techniques, and Applications, Birkhauser, Boston, 2007.

[18] N.G. Hadjiconstantinou, G. A. Radtke, L.L. Baker, "On variance-reduced simulations of the Boltzmann transport equation for small-scale heat transfer applications", J. Heat Trans., 132, 112401, 2010.

[19] G. A. Radtke, Efficient Simulation of Molecular Gas Transport for Micro- and Nanoscale Applications, PhD Thesis, MIT, 2011. 